\title{
Bioterrorismo: capacitar para responder
}

\author{
Bioterrorism: empower to respond \\ Ana Paula Chein Bueno de Azevedo', Simone Cynamon Cohen', Telma Abdalla de Oliveira \\ Cardoso 1
}

DOI: $10.1590 / 0103-110420195313$

RESUMO A utilização de agentes biológicos com a finalidade de aterrorizar inimigos surgiu há muitos séculos. Essa ação foi popularizada como bioterrorismo. Ataques bioterroristas são perigosos devido ao envolvimento de diversos fatores, como a forma silenciosa na qual pode ocorrer, o tipo e a forma de transmissão do agente biológico utilizado, dependendo do objetivo do ataque. Para prevenção da contaminação por esses agentes, os profissionais que irão atuar no seu combate devem possuir conhecimentos a respeito dos mecanismos de ação e de disseminação desses patógenos, bem como a adequada forma de sua neutralização e eliminação; além de saber escolher os equipamentos de proteção, tanto de uso individual quanto coletivo, adequados. Diante disso, tais equipes de ação necessitam ser bem capacitadas e treinadas. $\mathrm{O}$ objetivo deste estudo foi analisar, na literatura, os modos de treinamentos existentes no mundo para os profissionais de primeira resposta aos ataques envolvendo agentes Químico, Biológico, Radiológico ou Nuclear (QBRN). A partir de uma revisão integrativa, encontraram-se três artigos, em um período de 20 anos. Conclui-se que são muito escassos os estudos nessa área e que mais pesquisas e treinamentos para este tipo de evento devem ser realizados no mundo inteiro.

PALAVRAS-CHAVE Bioterrorismo. Biossegurança. Bombeiros.

\begin{abstract}
The use of biological agents for the purpose of terrorizing enemies dates back many centuries. This terror action with the use of biological agents became popularized as Bioterrorism. Bioterrorist attacks are dangerous due to various factors involved, such as the silent way in which it may occur, or the type of biological agent that can be used depending on the purpose of the attack. To prevent contamination by these agents, the professionals who will act to combat these events should have knowledge about the varieties of mechanisms of actions and dissemination of the pathogens, as well the best way to neutralize and eliminate them; besides, they should have known how to choose appropriates protection equipment, both for individual and collective protection. Thus, it is necessary that such action teams be well empowered and trained. The purpose of this paper is to analyze, in the existing literature, what are the training modes in the world for first responders to attacks involving Chemical, Biological, Radiological or Nuclear (CBRN) agents. Thus, an integrative review was made based on 3 articles found in a period of 20 years. It

is concluded that studies in this field are minimals and that more research and training for this kind of event should be carried out worldwide.
\end{abstract}

1 Fundação Oswaldo Cruz (Fiocruz), Escola Nacional de Saúde Pública Sergio Arouca (Ensp) - Rio de Janeiro (RJ), Brasil. ana.paula.azevedoo@gmail. com
KEYWORDS Bioterrorism. Biosafety. Firefighters. 


\section{Introdução}

O uso de agentes biológicos como armas de guerra não é uma novidade da era moderna. Embora não seja fácil datar o início do uso de armas biológicas, evidências apontam que, na era pré-cristã, por volta de 300 a.C., os gregos usavam cadáveres de animais para contaminar os poços de água dos inimigos. Em um período posterior, durante a batalha de Tortona, na Itália, em 1155, corpos de soldados e animais mortos foram usados para contaminar poços de água pelas tropas do Imperador Barbarossa'. No século XIV, durante o cerco da cidade de Kaffa (agora Feodosiya, na Ucrânia) pelos tártaros, espalhou-se uma epidemia de peste quando os sitiantes catapultaram cadáveres de seus companheiros mortos dentro das muralhas da cidade de Kaffa ${ }^{2}$.

$\mathrm{Na}$ história mais recente, durante a guerra franco-indiana, em 1763, o exército britânico utilizou cobertores contaminados com varíola para presentear os nativos indígenas ${ }^{3}$.

Na segunda metade do século XIX, com o desenvolvimento e avanço da área da microbiologia, houve o aumento do interesse militar pela utilização dos agentes biológicos como armas bélicas ${ }^{4}$.

Durante a Primeira Guerra Mundial, o exército alemão infectou bovinos e equinos com Burkhordelia mallei e Bacillus anthracis com a intenção de exportá-los para a tropa inimiga ${ }^{5}$. No Japão, uma unidade militar especial, a Unidade 731, foi fundada para realizar experimentos infectando seres humanos vivos com diversos tipos de agentes biológicos. $\mathrm{Na}$ Segunda Guerra Mundial, o Japão lançou bombas recheadas de pulgas e moscas infectadas com cólera, febre tifoide e peste bubônica nos assentamentos chineses ${ }^{6}$.

É importante ressaltar que, conceitualmente, existem diferenças entre guerra biológica e bioterrorismo. Apesar de ambos os eventos ocorrerem por meio da liberação intencional de um agente biológico, na guerra biológica, tem-se como alvo primário os militares, visando à destruição em massa das forças inimigas. O bioterrorismo, por outro lado, tem como alvo uma população heterogênea, visando atingir diferentes extratos, para causar paralisia social por meio de terror, pânico, ansiedade, medo, confusão e insegurança $\mathrm{a}^{7-9}$.

Esses eventos só serão possíveis quando houver o envolvimento de um ou mais patógenos (vírus, bactérias, fungos ou toxinas de organismos vivos) e um veículo para a disseminação ${ }^{10-12}$. Ressalta-se que os agentes biológicos podem ser organismos geneticamente modificados para aumentar suas características e potencializar o poder do ataque bioterrorista. Esses episódios tornam-se ainda mais perigosos quando os alvos são seres humanos devido ao fato de que, normalmente, a liberação dos agentes dar-se-á de forma silenciosa, ou seja, sem que haja explosão de bombas ou qualquer outro tipo de artefato explosivo, o que torna difícil a detecção da ocorrência do evento e facilita a disseminação dos agentes biológicos ${ }^{9}$.

Fatores como patogenicidade, alteração genética, resistência a drogas, modo de transmissão, endemicidade, disponibilidade de medidas terapêuticas e profiláticas, entre outras, são considerados na escolha do agente biológico. Todavia, os patógenos também podem ser escolhidos de acordo com o objetivo do ataque, ou seja, causar pânico ou incerteza na população, ou causar altas taxas de letalidade ou de morbidade, ou até mesmo pelo seu potencial de causar danos econômicos ${ }^{\mathbf{1 3}, 14}$.

A avaliação de risco dos agentes biológicos, sob o enfoque da biossegurança, é necessária para a determinação do que se considera risco, seu potencial, determinação das causas e medidas a serem tomadas para minimizá-lo, eliminá-lo ou controlá-lo. Essas medidas são conhecidas como barreiras de contenção, que podem ser primárias e secundárias. As barreiras de contenção primárias visam proteger os profissionais contra a exposição aos agentes de risco por meio da utilização de procedimentos e de Equipamentos de Proteção: Individual (EPI) e Coletiva (EPC) ${ }^{\mathbf{1 5}}$. Já as barreiras de contenção secundárias estão relacionadas com às características ambientais, construtivas e 
arquitetônicas dos locais de trabalho, e irão variar dependendo do risco de transmissão e de disseminação dos agentes que serão manipulados em cada espaço.

O Centers for Disease Control and Prevention (CDC) classifica os agentes biológicos em três categorias (A, B e C), de acordo com critérios que determinam a potencialidade de seu uso como armas biológicas ${ }^{16}$. Na categoria A, estão os agentes biológicos que são facilmente disseminados, causam altas taxas de letalidade e geram grande impacto à saúde pública, como varíola major, Bacillus anthracis, Yersinia pestis, Clostridium botulinum e Francisella tularensis. Na categoria B, estão os agentes biológicos de fácil disseminação, com taxa de morbidade moderada e baixa taxa de letalidade, como Coxiella burnetti, Salmonella spp., Shigella dysenteriae, Escherichia coli e Vibrio cholerae. Já na categoria C estão os agentes emergentes, que podem ser manipulados por meio da engenharia genética, podendo ser facilmente obtidos, produzidos e disseminados, possuindo altas taxas de morbidade, letalidade e potencial para causar grandes impactos nos sistemas de saúde, como o vírus Nipah, Hantavírus, vírus da Febre Amarela e Mycobacterium tuberculosis resistente a múltiplas drogas.

Quando há casos de suspeita de um evento Químico, Biológico, Radiológico ou Nuclear (QBRN), várias agências serão acionadas dentro do seu nível de competência, com o intuito de preservar a saúde e a segurança dos envolvidos no evento ${ }^{17}$, evitando agravamento da situação. Os profissionais dessas agências são da área da saúde, como médicos e enfermeiros, profissionais de defesa civil, bombeiros militares, militares das forças armadas, polícia federal entre outros ${ }^{\mathbf{1 8}}$.

Os bombeiros realizam a primeira resposta, com o resgate das vítimas, isolamento da área afetada, identificação do local atingido e descontaminação dessa região ${ }^{19}$. Esses procedimentos são realizados mediante o estabelecimento de quatro perímetros, que irão auxiliar na contenção e proteção necessários.
Esses perímetros são denominados: a) zona quente, área onde o evento ocorreu e de máxima contaminação; b) zona morna, área de transição, onde ocorrem os procedimentos de descontaminação; c) zona fria, área livre de contaminação, onde ficam localizados o posto de comando da operação e outras áreas de suporte e logística; e d) zona de exclusão, área onde devem permanecer pessoas e instituições que não estão envolvidas com o ocorrido ${ }^{\mathbf{2 0}-22}$.

A discussão a respeito do treinamento adequado dos profissionais de primeira resposta ainda é pouco realizada. Não há um plano de ação único, em que estejam descritos os procedimentos de atuação dos profissionais de primeira resposta QBRN principalmente relacionados com o bioterrorismo, que, entre os eventos QBRN, é o mais difícil de detecção.

Destarte, o objetivo deste estudo é analisar o que tem sido publicado nas últimas décadas em relação aos modelos de treinamentos dos profissionais de primeira resposta para eventos de bioterrorismo.

\section{Material e métodos}

Este é um estudo exploratório descritivo, valorizando a revisão integrativa. Esse método busca revisar, sistematizar e analisar criticamente a literatura científica relacionada a um determinado tema, de modo sistemático ou ordenado, gerando novas perspectivas. $\mathrm{Ou}$ seja, busca construir uma análise sobre o que é conhecido na literatura, baseado em pesquisas anteriores, sobre o tema em questão, descrevendo as características de determinada população ou fenômeno ou, estabelecendo relações entre variáveis, auxiliando assim, na geração de conhecimentos ou na resolução de um problema ${ }^{23-27}$.

A questão norteadora da pesquisa foi: quais os aspectos metodológicos utilizados nos treinamentos para eventos de bioterrorismo dos profissionais de primeira resposta?

Para a seleção dos artigos, foram utilizadas as seguintes bases de dados: Medical Literature 
Analysis and Retrieval System Online (Medline/Pubmed), Índice Bibliográfico Español de Ciencias de la Salud (Ibecs), Literatura Latino-Americana e do Caribe em Ciências da Saúde (Lilacs), Cochrane e Scientific Eletronic Library Online (SciELO).

Foram utilizados os vocabulários estruturados empregados tanto pela Biblioteca Virtual em Saúde, os Descritores em Ciências da Saúde (DeCS); quanto pela United States National Library of Medicine (NLM), os descritores Medical Subject Headings Section (MESH). Utilizaram-se os operadores booleanos $O R$ e $A N D$ (quadro 1). A busca foi realizada no período de 1999 a 2019, tendo sido executada no dia 25 de março de 2019.

Quadro 1. Estratégias de busca

\begin{tabular}{ll}
\hline Bases de dados & Estratégias de busca \\
\hline Lilacs & (Bioterrorismo OR Terrorismo biológico OR Bioterrorism) AND (Bombeiros OR Polícia e Bombeiros \\
Ibecs & em Desastres OR Firemen and Policemen in Disasters OR Firefighters) \\
Cochrane & (Bioterrorismo OR bioterrorism) AND (Bombeiros OR Firefighters) \\
SciELO & (Biossegurança OR Containment of Biohazards OR Biosafety) AND (Bioterrorismo OR Bioterro- \\
& rism) AND (Bombeiros OR Firefighters) \\
& (Biossegurança OR Contenção de risco biológico OR Containment of Biohazards OR biosafety) \\
& AND (Bombeiros OR Polícia e Bombeiros em Desastres OR Firemen and Policemen in Disasters OR \\
& Firefighters) \\
& (Bioterrorismo OR Terrorismo biológico OR Bioterrorism OR Guerra Biológica OR Biological Warfa- \\
& re OR Armas Biológicas OR Biological Warfare Agents) AND (Bombeiros OR Firefighters) \\
& (Bioterrorismo OR Terrorismo biológico OR Bioterrorism OR Guerra Biológica OR Biological Warfa- \\
& re OR Armas Biológicas OR Biological Warfare Agents OR Biological Weapon OR Biological terro- \\
& rism OR Biowarfare) AND (Bombeiros OR Firefighters) \\
& Bioterrorism AND (Firemen and Policemen in Disasters OR Firefighters) \\
& Bioterrorism AND Firefighter \\
& (Bioterrorism OR Biological Warfare OR Biological Warfare Agents) AND (Firemen and Policemen \\
in Disasters OR Firefighters) & (Biological Warfare OR Biological Warfare Agents OR Biological weapon OR Biological terrorism) \\
Medline & AND (Firemen and Policemen in Disasters OR Firefighters) \\
\hline
\end{tabular}

Fonte: Elaboração própria.

Para a seleção dos artigos, estabeleceram-se como critérios de inclusão: a) período de publicação; b) somente artigos; c) publicação nas línguas inglesa, portuguesa ou espanhola; e d) publicações disponibilizadas na íntegra. Como critérios de exclusão, adotaram-se: a) editoriais, cartas ao editor, resumos, comentários e notas prévias; b) publicações sem a especificidade de treinamentos para profissionais de primeira aos eventos de bioterrorismo; c) artigos com conteúdo de caráter geral; e d) artigos duplicados.

A primeira seleção foi realizada a partir da leitura dos títulos e resumos, de acordo com os critérios de inclusão e exclusão, que orientou a seleção dos artigos para leitura integral e análise dos textos.

\section{Resultados e discussão}

Encontraram-se 63 resultados nas bases de dados pesquisadas. Para a elegibilidade, foram analisados os critérios de inclusão no estudo. Por fim, ao serem retiradas as duplicadas, totalizaram três artigos a serem analisados (figura 1). 
Figura 1. Resultados encontrados

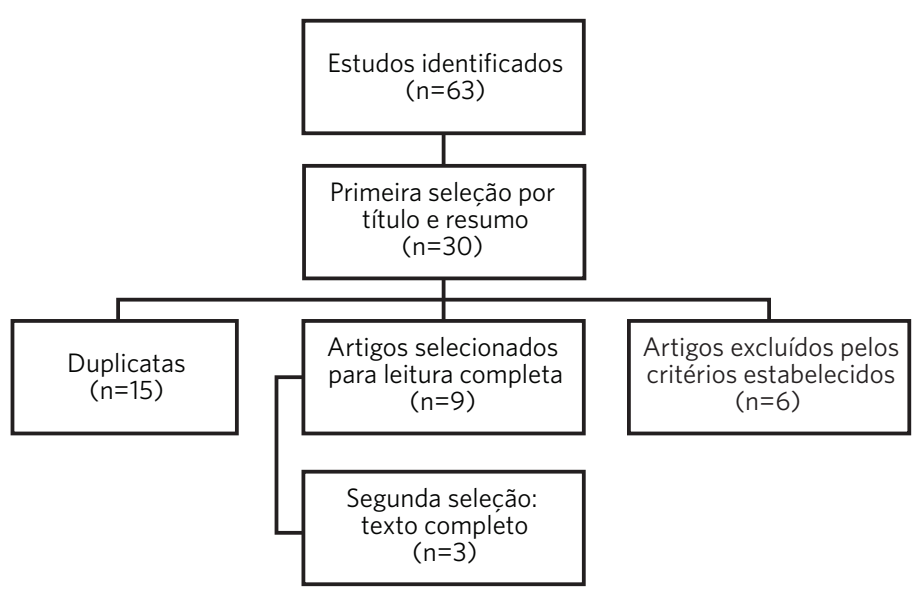

Fonte: Elaboração própria.

Cada artigo identificado analisa o treinamento desses profissionais em um país (Estados Unidos, Alemanha e Reino Unido). Os autores dos artigos identificados, diante de diferentes circunstâncias, demonstraram que os profissionais responsáveis pela defesa civil em casos de eventos QBRN, sobretudo no bioterrorismo, não possuíam o treinamento adequado para responderem aos eventos. A partir desse despreparo, os governos locais de cada país começaram a desenvolver um planejamento e treinamento das equipes responsáveis por atender às emergências QBRN; e, por fim, verificou-se a eficácia desses planos.

O objetivo do estudo de Beaton e Johnson ${ }^{\mathbf{2 8}}$ foi testar a eficiência de um treinamento de preparo doméstico. Nesse estudo, participaram 610 profissionais de defesa civil dos Estados Unidos, divididos em quatro grupos: pré-treino, pós-treino, com treinamento doméstico e sem treinamento doméstico.

Com o intuito de descrever com realismo, foi feito um questionário para analisar a preparação doméstica e o conhecimento necessário para a operação de primeira resposta aos eventos. Treinamentos de 30 horas foram realizados, nos quais se avaliaram os resultados.
Obtidos antes e após o treinamento, os resultados demonstraram que é necessária a preparação para ataques terroristas envolvendo armas de destruição em massa e a importância desse treinamento.

Dessa forma, Beaton e Johnson ${ }^{\mathbf{2 8}}$ relatam que o Departamento de Defesa dos Estados Unidos, juntamente com outras agências governamentais americanas, criou esforços para providenciar um plano de preparo de primeiras respostas em centros urbanos voltados para ameaças químicas, biológicas e nucleares, ou que envolvam qualquer armamento de destruição em massa. $\mathrm{O}$ objetivo desse planejamento é o treinamento, educando cada profissional responsável pela primeira resposta nos centros urbanos, para que saibam responder a cada situação, especificamente reconhecendo sinais e sintomas de um ataque de uma arma de destruição em massa, identificando e implementando a resposta adequada para cada tipo de situação enfrentada.

O estudo de Lenz e Richter ${ }^{29}$ teve como objetivo desenvolver e aplicar um instrumento de avaliação do desempenho dos profissionais do Corpo de Bombeiros da Alemanha durante exercícios simulados de bioterrorismo. 
Houve a participação de 68 profissionais. Das 31 atividades realizadas nos exercícios, 20 foram executadas corretamente, e 1 não era aplicável ao contexto. Foram identificados problemas relativos à identificação e ao manuseio de materiais perigosos, à utilização de equipamentos de proteção e ao processo de descontaminação; demonstrando falta de treinamento adequado e problemas de comunicação e comando.

Com isso, Lenz e Richter ${ }^{29}$ concluíram que é necessário mais treinamento para resposta aos incidentes com materiais biológicos, além de reavaliar constantemente seus procedimentos técnicos e habilidades específicas, bem como suas estruturas de comunicação e comando. Ademais, os autores também apontam para a necessidade de mais pesquisas relacionadas com o assunto e com o desenvolvimento de um sistema de avaliação que meça melhor os exercícios em escala real.

O estudo de Holdsworth, Bland e O'Reilly ${ }^{30}$ discutiu a preparação tanto civil quanto militar em relação às emergências relacionadas com as ameaças QBRN. Tal discussão concentrou-se na preparação e na capacidade de resposta dos serviços de Defesa e Emergência do Reino Unido em cenários que poderiam ocorrer eventos QBRN, além de debaterem também os desafios futuros a serem enfrentados.

Os autores ressaltam que as ações em resposta às ameaças QBRN devem ser desenvolvidas em quatro áreas: prevenção, preparação, resposta e recuperação. No entanto, a experiência demonstra que ações na fase de recuperação de um incidente QBRN de grande escala, em tempo de paz, são muito limitadas. Enfatizam que a preparação para essa função é essencial para mitigar o objetivo dos terroristas; defendendo a necessidade de exercícios de treinamento em todos os níveis de resposta QBRN. Analisando todas as etapas dos exercícios, Holdsworth, Bland e O'Reilly ${ }^{30}$ concluíram que existem desafios nas equipes de resposta relacionados com a falta de treinamento e do uso de equipamentos adequados.

\section{Conclusões}

Nos artigos analisados, observou-se que o tema bioterrorismo continua a ser uma ameaça credível, com um impacto potencialmente catastrófico. No Brasil, o Corpo de Bombeiros é responsável pelos primeiros atendimentos em um evento QBRN, porém, seu corpo técnico possui limitações em um possível grande evento. O problema envolvendo conhecimento, treinamento e técnica no que concerne aos eventos bioterroristas não é uma lacuna somente para o Brasil. Grandes nações, como Estados Unidos, Alemanha e Reino Unido, também lidam com dificuldades em relação ao tema de bioterrorismo, devido ao fato desse tipo de evento QBRN não ser tão facilmente identificado nos anos passados, o que leva a crer que é um evento com pouca probabilidade de ocorrência, o que de fato não é verdade.

Com o aumento da possibilidade de uso de armas biológicas para a realização de ataques bioterroristas, devido ao desenvolvimento de novas tecnologias e de novas armas de destruição em massa, com maior grau de letalidade e menor capacidade de rastreamento; as armas biológicas poderão ser utilizadas no lugar de outras armas não convencionais (como as armas químicas ou nucleares), o que faz com que o conhecimento a respeito de bioterrorismo ganhe destaque e necessite ser mais socializado.

O gerenciamento imediato do cenário, a liberação, o estabelecimento e a manutenção de um cordão de isolamento e o gerenciamento das vítimas são desafios significativos. A exigência de descontaminação em massa de um grande número de pessoas poderia aumentar rapidamente e precisa ser considerada. Para tanto, há de ter condições técnico-operacionais para isso, além de cuidados preventivos para evitar a contaminação de outros indivíduos e do meio ambiente. O entendimento e a comunicação interagências, nacionais e internacionais, são essenciais para otimizar a resposta e aumentar a capacidade de resposta.

Os profissionais de primeira resposta 
precisam estar capacitados; para isso, é necessário que estudos e treinamentos sejam realizados e avaliados para que se possa determinar os pontos a serem reforçados na capacitação desses profissionais, visando ao aperfeiçoamento para garantir a própria segurança e da população a que estão protegendo.

O desenvolvimento de uma doutrina clara e de procedimentos operacionais padrão seguidos de exercícios, envolvendo todas as organizações de resposta para a prática de cenários de resposta, é fundamental para alcançar um estado de prontidão.

Por meio dos resultados desta pesquisa, pode-se perceber também a escassez de estudos e publicações quanto a essa temática, o que a torna um assunto de grande importância e que necessita de maior visibilidade.

\section{Colaboradores}

Azevedo APCB (0000-0003-4202-4919)*, Cohen SC (0000-0001-6228-6583)* e Cardoso TAO (0000-0002-5430-7273)* contribuíram igualmente para a concepção, levantamento de dados, elaboração do rascunho e da redação, revisão crítica do conteúdo e aprovação da versão final do manuscrito.

\section{Referências}

1. Clarke R. The Silent Weapons. New York: David McKay Co; 1968.

2. Wheelis M. Biological Warfare at the 1346 Siege of Caffa. Emerg Infect Dis. 2002; 8(9):971-5.

3. Bhalla D, Warheith D. Biological agents with potential for misuse: ahistorical perspective and defense measures. Toxicol Appl Pharm. 2004; 199(1):71-84.

4. Davison N. The Role of Scientific Discovery in the Establishment of the First Biological Weapons Programmes. Bradford Scien. and Tec. Report. 2005; (5):1-27.
5. Redmond C, Pearce M, Manchee R. Deadly relic of the Great war. Nature. 1998; 393(6687):747-8.

6. Harris S. Factories of Death: Japanese Biological Warfare, 1932-1945, and the American Cover-Up. London: Routledge; 2002.

7. Cardoso DR, Cardoso TAO. Bioterrorismo: dados de uma história recente de riscos e incertezas. Ciênc. Saúde Colet. 2011; 16(1):3129-38.

8. Grayson ML. The difference between biological warfare and bioterrorism: Australia finally makes a start towards real preparedness for bioterrorism: Biolo-
${ }^{*}$ Orcid (Open Researcher and Contributor ID). 
gical warfare and bioterrorism. Intern Med J. 2003; 33(5-6):213-4

9. Rebmann T. Infectious Disease Disasters: Bioterrorism, Emerging Infections, and Pandemics. In: Association for Professionals in Infection Control and epidemiology, organization. Text of infection control \& epidemiology. Arlington: APIC; 2014. Chapter 120. p. 1-22.

10. Tucker JB. The Current Bioweapons Threat. In: Hunger I, Radosavljevic V, Belojevic G, et al., organizadores. Biopreparedness and $\mathrm{Pu}$ blic Health [internet]. Dordrecht: Springer Netherlands; 2013. p. 7-16. [acesso em 2018 out 21]. Disponível em: http://www.springerlink.com/index/10.1007/978-94-007-5273-3_2.

11. Vogel KM. Phantom menace or looming danger? a new framework for assessing bioweapons threats. Baltimore: Johns Hopkins University Press; 2013.

12. Zalini Y. Combating and reducing the risk of biological threats. J Def Secur. 2010; 1(1):1-12.

13. Pohanka M, Kuca K. Biological warfare agents. Mol Clin Enrivonmental Toxicol. 2010; 100(2):559-60.

14. Tucker JB. Historical trends related to bioterrorism: An empirical analysis. Emerg Infect Dis. 1999; 5(4):498-504

15. Rocha SS. Conceitos Básicos em Biossegurança. In: Oda LM, Ávila SM, organizadores. Biossegurança Em Laboratórios de Saúde Pública. Brasília, DF: MS; 1998. p. 15-30.

16. Center for Diseases Control and Prevention. Emerging Infectious Diseases Related to Travel. In: CDC Yellow Book. New York: Oxfor University Press; 2018. Chapter 3. p. 139-424.

17. Rambauske D, Cardoso TAO, Navarro MBMA. Bioterrorismo, riscos biológicos e as medidas de biossegurança aplicáveis ao Brasil. Rev. Saúde Colet. 2014; 24(4):1181-205.
18. Pires LAA, Vasconcellos LCF, Bonfatti RJ. Bombeiros militares do Rio de Janeiro: uma análise dos impactos das suas atividades de trabalho sobre sua saúde. Saúde debate. 2017; 41(113):577-90.

19. Brasil. Ministério da Saúde. Classificação de Risco dos Agentes Biológicos. 3. ed. Brasília, DF: Ministério da Saúde; 2017.

20. Brasil. Ministério da Saúde. Plano de Contingência para Emergências em Saúde Pública por Agentes Químico, Biológico, Radiológico e Nuclear. Brasília, DF: Ministério da Saúde; 2014.

21. Canadá. Cheminal, Biological, Radiological, Nuclear First Responder Training Program. Basci Level Pre-Course Reading. Ontario: Canadian Emergency Management College; 2018.

22. Reino Unido. Department for Communities and Local Government. Fire and rescue service operational guidance: incidents involving hazardous materials. Londres: Department for Communities and Local Government; 2012.

23. Brosseau L, Laroche C, Guitard P. The French-Canadian Version of the Assessment of Multiple Systematic Reviews (AMSTAR) Tool. Physioth. Canada. 2017; 69(1):20-9.

24. Whitemore R, Knafl K. The integrative review: updated methodology. Blackwell Publishing Ltd. 2005; 52(5):546-53.

25. Mendes KD, Silveira RCC, Galvão C. Revisão integrativa: método de pesquisa para a incorporação de evidências na saúde e na enfermagem. Texto contexto- enferm. 2008; 17(4):758-64.

26. Benefield L. Implementing evidence-based practice in home care. Home healthc. nurse. 2003; 21(12):804-11.

27. Polit DF, Beck CT. The content validity index: Are you sure you know what's being reported? critique and recommendations. Res Nurs Health. 2006; 29(5):489-97. 
28. Beatons R, Johnson C. Instrument Development and Evaluation of Domestic Preparedness Training for First Responders. Prehosp Disaster Med. 2002; 17(3):113-25.

29. Lenz M, Richter T. Disaster Response to the Release of Bioharzardous Agents: Instrument Development and Evaluation of a Firefighte's Exercise. Prehosp Disaster Med. 2009; 24(3):197-205.
30. Holdsworth D, Bland S, O'Reilly D. CBRN Response and the Future. J R Army Med Corps. 2012; (158):58-63.

Recebido em 30/04/2019

Aprovado em 21/08/2019

Conflito de interesses: inexistente

Suporte financeiro: não houve 